\title{
UTILIZAÇÃO DE INDICADORES DE DESEMPENHO NO DESENVOLVIMENTO ESTRATÉGICO LOGÍSTICO EM EMPRESAS DE TRANSPORTE RODOVIÁRIO TERCEIRIZADO
}

\author{
USE OF PERFORMANCE INDICATORS IN THE STRATEGIC LOGISTIC \\ DEVELOPMENT IN OUTSOURCED ROAD TRANSPORTATION COMPANIES
}

\author{
Karla Raquel Rosa - k.r.rosa@outlook.com \\ Paulo Henrique Benedeti - paulohbenedeti@gmail.com \\ Oswaldo Lázaro Mendes - oswaldo.lazaro@fatectq.edu.br \\ Faculdade de Tecnologia de Bebedouro (FATEC) - SP - Brasil
}

DOI: 10.31510/infa.v15i1.370

\section{RESUMO}

O cenário de mercado altamente competitivo e avanços tecnológicos impulsionaram a busca de gestores por eficiência em processos que assegurem o bom desempenho e qualidade das atividades desenvolvidas. O objetivo desse artigo é identificar como o uso de indicadores de desempenho pode auxiliar no desenvolvimento estratégico da logística empresarial e contribuir para a garantia da eficiência e da qualidade dos serviços de transporte terceirizados. Por meio de estudos e levantamento bibliográfico busca-se demonstrar que os indicadores de desempenho podem ser uma ferramenta estratégica para o aumento da eficiência em serviços logísticos e qualidade de transporte terceirizado. Os resultados obtidos são satisfatórios, considerando que o problema de pesquisa foi respondido e os objetivos alcançados. Pois, o uso de indicadores de desempenho são uma excelente ferramenta estratégica e vem sendo incorporada por diversos autores e empresas, uma vez que ela pode proporcionar a elaboração de planos de melhoria contínua em busca de oferecer ao mercado e ao cliente um alto nível de serviço e garantir a qualidade em seus processos. Os resultados servirão como base para estudo futuro que assumirá a forma de estudo de caso. Será realizado o levantamento de dados reais que permitirão a comparação entre teoria e prática.

Palavras-chave: Logística. Transporte. Indicadores de desempenho.

\begin{abstract}
The current scenario of a highly competitive market and technological advances impelled managers to search for efficiency in processes that ensure the good performance and quality of the developed activities. The objective of this article is to identify how the use of performance indicators can assist in the strategic development of business logistics and contribute to guarantee the efficiency and quality of outsourced transportation services. Through reading and bibliographical research, it is sought to demonstrate that performance indicators can be a strategic tool in order to improve the efficiency in logistics services and quality of outsourced transportation. The results obtained are satisfactory, since the research problem was solved, and the objectives were achieved. It was possible to attest that the use of performance indicators is an excellent strategic tool and that it has been incorporated by several authors and companies, since it helps to elaborate plans for continuous improvement
\end{abstract}


in order to offer a high level of service to customers and to the market, as well as ensure quality in their processes. The results will serve as a basis for a future study that will take the form of a case study. The collecting of real data will lead to a comparison between theory and practice.

Keywords: Logistics. Transport. Performance indicators.

\section{INTRODUÇÃO}

A competitividade entre mercados aguçou a preocupação, dos empreendedores, quanto aos clientes, aos concorrentes e ao grande número de varáveis que sofrem constante mudança no Brasil e no mundo empresarial (MAXIMIANO, 2008). Agregar valor há produtos e serviços é vista, atualmente, como ferramenta fundamental na obtenção de vantagem competitiva (BALLOU, 2006).

Em uma visão moderna de gestão, pode-se ententer que a empresa é um sistema integrado por vários precessos e subprocessos que trabalham juntos para alcançar os objetivos estabelecidos (MAXIMIANO, 2008). Ballou (2006), destaca a logística como parte desse “sistema”, sendo vista hoje, de forma integrada a gestão juntamente com as demais áreas.

Avanços em conceitos logísticos dentro e fora das organizações apresentam crescimento e atualização de maneira acelerada nos últimos anos. No ano de 1970, os cursos de Engenharia de Produção, começaram a capacitar profissionais que posteriormente foram atuar em logística. Atualmente novos cursos vêm sendo inseridos no contexto educacional, abrindo as "portas" das empresas que buscam desenvolvimento e estruturação da logística em suas atividades (DIAS, 2017).

Planejar estrategicamente os processos faz parte da gestão empresarial e a medição sistemática de desempenho, pode garantir o sucesso das ações excutadas. E a aplicabilidade de indicadores possibilita o monitoramento da performace em determinado processo, ou até mesmo, de um processo dentro do sistema, cria uma linguagem e alinhamento dos objetivos e metas (KAPLAN; NORTON, 1997).

Quando se fala em estratégia, o termo "indicadores de desempenho" pode ser efetivo, pois fomentam embasamento na escolha eficaz de metodologias de ação e gerenciamento sendo importante na manutenção da integração dos elos da cadeia (MAZZA; CORREIA, 2016). E com relação ao atual cenário, é preciso refletir sobre a infraestrutura brasileira e os 


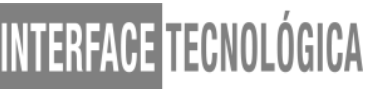

problemas logístico atuais encontrados no país, principalmente relacionados a altos custos e precariedade de modais para transporte.

O objetivo desse artigo é identificar como o uso de indicadores de desempenho pode auxiliar no desenvolvimento estratégico da logística empresarial. Além de contribuir para a busca por eficiência como ferramenta competitiva. E tratando-se de eficiência, verificar se pode ser estendido o uso de indicadores como ferramenta na gestão da qualidade no transporte terceirizado. Através de estudos e levantamento baseados em revisão bibliográfica busca-se resposta para o problema: o uso de indicadores de desempenho pode ser uma ferramenta estratégica na busca por eficiência em serviços logísticos e qualidade de transporte terceirizado?

A abordagem do artigo, direcionada para transporte terceirizado, dá-se pela importância da distribuição e a movimentação de materiais e produtos dentro da cadeia de suprimentos. No mapeamento estratégico torna-se um processo chave para acrescer a participação no mercado e consequentemente o aumento da lucratividade, desde pequenas a grandes organizações (DIAS, 2017). E é relevante o fato de que nos últimos anos, a terceirização em serviços logísticos, torna-se uma alternativa na busca pela eficiência em processos.

\section{REVISÃO DA LITERATURA}

A logística é considerada um conceito moderno, apesar de ser identificada como uma atividade econômica antiga. A evolução da produtividade proporcionou resultados excedentes a demanda necessária para consumo imediato, surge então a ideia de estocagem. $\mathrm{O}$ transporte foi praticamente a consequência da necessidade de movimentação para que a produção fosse comercializada (DIAS, 2017).

Ainda Dias (2017), cita a definição de logística da Council of Supply Chain Management Professionals:

“A logística planeja, executa, coordena e controla a movimentação e o armazenamento eficiente e econômico de matérias-primas, materiais semiacabados e produtos acabados, desde sua origem até o local de consumo, com o propósito de atender às exigências do cliente final". (DIAS, 2017, p. 3) 


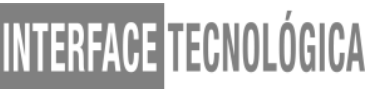

Seguindo citações na obra de Dias (2017), para a Associação Brasileira de Logística (ABRALOG) e a Associação Brasileira de Logística e Transporte de Cargas (ABTC), a logística é responsável por planejar, implantar e controlar com eficiência o fluxo da cadeia.

O sistema deve ainda, abranger conceitos de disponibilidade, desempenho operacional e confiabilidade do serviço, e seus resultados devem ser metodicamente medidos. (FLEURY; WANKE e FIGUEIREDO, 2000). Buscando atender os principais objetivos estratégicos da logística: custos baixos, redução de necessidade de capital e melhorias para assegurar a qualidade no serviço (BALLOU, 2006).

\subsection{Distribuição e terceirização de transporte}

O processo de desenvolvimento de estratégias que garantam eficiência e qualidade na distribuição de materiais e produtos são fundamentais para garantir a competitividade no mercado. O processo de distribuição está ligado a movimentação do material de um ponto da produção/armazenagem até o cliente final, e faz parte das atividades da administração de materiais (GONÇALVES, 2013).

As atividades internas, serviço ao cliente e o levantamento de informações dentro do planejamento estratégico, busca otimizar a cadeia de Supply Chain e agregar, a projetos logísticos, vantagem competitiva em ternos de serviços e velocidade de retorno do mercado (BALLOU, 2006).

Movimentar produtos, escolher o melhor roteiro e a melhor utilização da capacidade de transporte, assim como, controlar os custos envolvidos, são atividades que correspondem a gestão de transporte. As decisões relacionadas ao planejamento de transporte impactam de forma direta na redução de custos, economia de escala e na garantia de nível de serviço com qualidade (DIAS, 2017).

O Brasil ainda concentra nas rodovias a maior parte do transporte de cargas, e uma das consequências foi o crescimento ágil e desproporcional do modal rodoviário, comparado aos demais existentes (BALLOU, 2006).

Ainda Dias (2017), o índice 55\% do volume total de carga movimentada no país está nas mãos de carreteiros e um dado interessante sobre a terceirização é que apenas $3,5 \%$ das 3.500 empresas cadastradas na Agência Nacional de Transportes Terrestres (ANTT) possuem frota própria. A participação dos serviços logísticos de transporte no Produto Interno Bruto (PIB) é de 7\%, sendo 3,5\% oriundas de serviços prestados por carreteiros. 


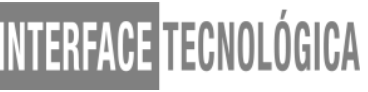

Conhecidos como Prestadores de Serviços Logísticos (PSLs), os fornecedores de serviços especializados em logística, ofertam serviços desde as formas mais simples e mais tradicionais de atividades como o transporte, estocagem e armazenagem. Os PSLs apresentam baixo custo de aquisição/investimento em capital para o contratante do serviço, fato que influenciou no aumento das decisões relacionadas à adoção desse modelo de serviço no Brasil e no mundo (BARROS, 2009).

\subsection{Indicadores de desempenho}

De acordo com Sucena e Silva apud Belém Jr. (2013) o ato de medir algo é uma junção de atividades desempenhadas, seus pressupostos e técnicas utilizadas, tendo como principal objetivo, quantificar variáveis e propriedades da instância que se irá avaliar. Portanto faz-se necessário a compreensão, de forma clara e objetiva, das medidas adotadas e para qual ação devem ser direcionadas pela empresa, no intuito de almejar melhoria das atividades desenvolvidas. Em outras palavras pode-se entender que os indicadores de desempenho são uma ferramenta para se analisar o cumprimento dos objetivos antemão traçados no planejamento estratégico, pois auxiliam no monitoramento das atividades que agregam valor a empresa (SUCENA; SILVA, apud ANGÊLO, 2013).

Formando-se um conjunto de objetivos e indicadores, que se inter-relacionem, é possível projetar os resultados em forma de diagrama de causa e efeito (MAXIMIANO, 2008).

Sucena e Silva (2013), levantam em seu estudo publicado no IX Congresso Nacional de Excelência em Gestão, 2013, os autores de maior destaque pela contenção na especificação de medidas de desempenho em transporte rodoviário de cargas. Segue conclusões consideradas fundamentais para o presente estudo:

Ainda Sucena e Silva apud Amancio et al. (2013), busca em seu estudo, junto ao operador logístico, identificar as medidas necessárias de avaliação de desempenho. A conclusão dos autores após a análise dos fatos é que a necessidade de implementação de indicadores tem extrema importância na medição e controle dos processos gerenciais, destacando o transporte de carga entre eles. Também concluem ser básica a atenção para mensuração de avarias e perdas. E considerando as questões estratégicas, a terceirização de operações logísticas pode ser efetiva, assim como no âmbito operacional. 


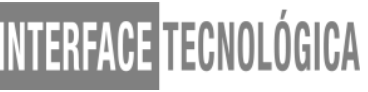

Em outro estudo, Sucena e Silva apud Oliveira Neto et al. (2013), averiguaram a importância da implementação de indicadores de desempenho como forma de controle com a finalidade de avaliar os serviços logísticos de transporte rodoviário de cargas terceirizado. Completando Sucena e Silva apud Siluk et al. (2013), após análise desenvolvida em seu trabalho, propõe um procedimento de avaliação do desempenho específico para empresas de transporte rodoviário de cargas e o autor Ângelo (2005), sugere os principais indicadores a serem utilizados, dividindo-os em: atendimento ao cliente, gestão de estoques, armazenagem e gestão de transportes.

\subsection{Eficiência e qualidade na medição de desempenho}

Buarque et al. (2003), afirma que a eficiente medição de desempenho propicia o controle satisfatório das atividades operacionais da empresa, auxilia na desenvolvimento da criação e implantação de medidas estratégicas, e conduz tais estratégias de forma a tornaremse diferencial competitivo. Contribui também, na indicação de falhas no sistema e na identificação da necessidade de atuação da gestão.

A definição dos indicadores a serem implantados em um plano estratégico é fundamental para garantir o desempenho correspondente ao seu uso. O Balanced ScoreCard (BSC) ou Indicadores de Desempenho Organizacional, é uma metodologia que visa a integração e o balanceamento de todos os indicadores quem podem ser aplicados em uma organização, através de metas claramente estabelecidas (KAPLAN; NORTON, 1997). Seguindo Kaplan e Norton (1997), os indicadores dentro do BSC se dividem em quatro perspectivas: financeira; clientes e mercado; processos e operações internas; inovação, aprendizado e sustentabilidade. 
Figura 1. BSC - conectando indicadores à estratégia

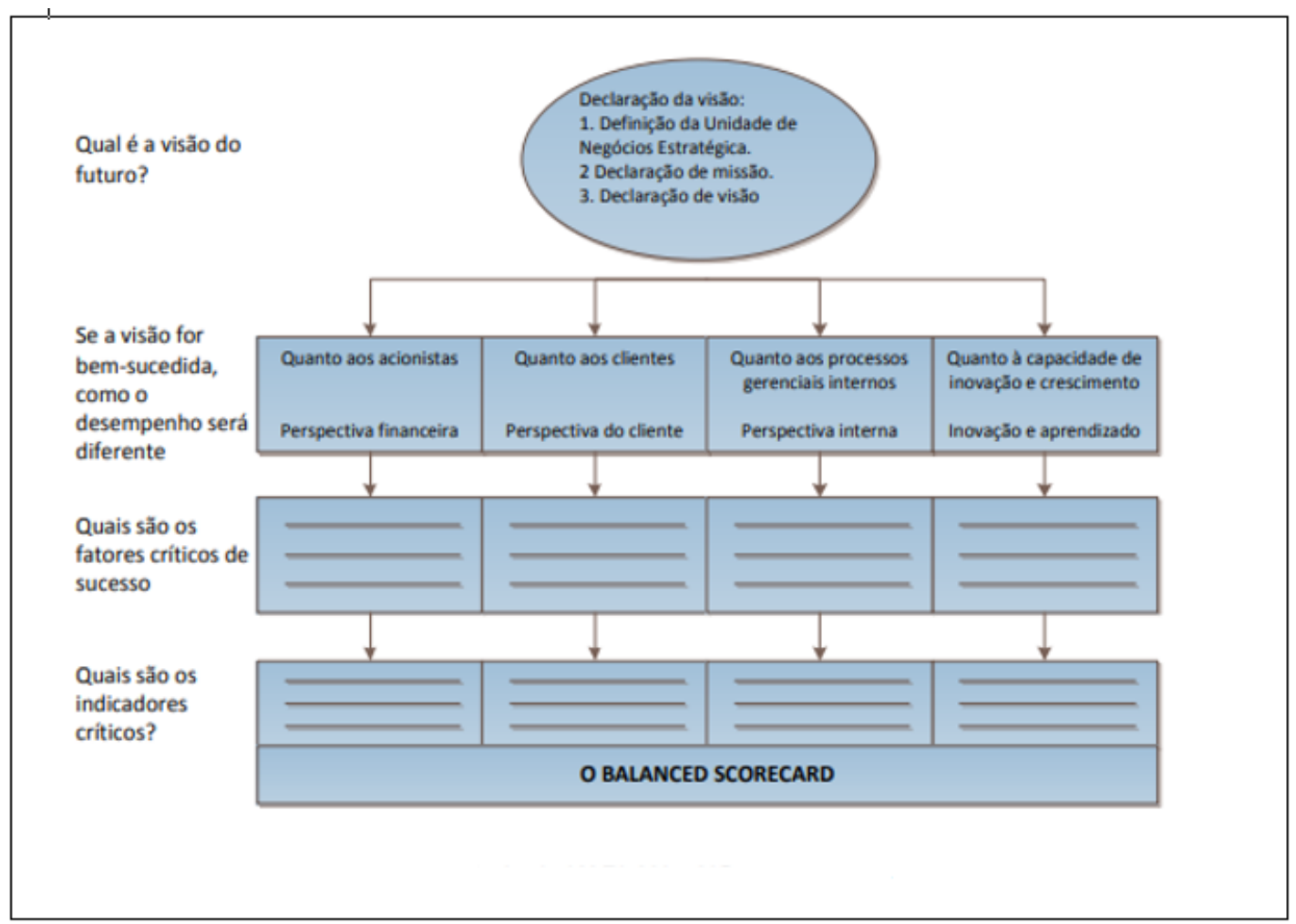

Fonte: adaptado de Kaplan e Norton (1993) por: Shiro (2011).

Do ponto de vista de Bowersox, Closs e Cooper (2007), para que o sistema de gestão estratégia através de indicadores seja eficiente, é preciso medir não apenas o funcionamento do sistema, mas integrar as informações a cadeia de suprimentos e ao nível de atendimento oferecido ao cliente. São quatro as principais categorias de medidas de desempenho logístico:

- Custos: o desempenho logístico tem impacto direto nos custos e esses, devem ter seu desempenho mesurado;

- Serviço ao cliente: deve-se acompanhar o desempenho do serviço através de métricas de avaliação da disponibilidade, do desempenho operacional e da confiabilidade do serviço;

- Qualidade: monitoramento da eficácia das atividades, pode basear-se em número de reclamações ou devoluções, entre outros;

- $\quad$ Produtividade: expõe a analogia entre a saída de produtos e a quantidade de recursos disponibilizados na produção;

- Gerenciamento de ativos: mensura como é utilizado, pelos gestores, os recursos financeiros disponíveis de capital investido. 
Alcançar eficiência e qualidade em serviços logísticos, pode assegurar a fidelização de clientes resultando em aumento de retenção de clientes e consequentemente, aumento da lucratividade (BALLOU, 2006).

Sucena e Silva (2013), apresentam como resultado de seus estudos, uma lista de indicadores baseada em autores citados anteriormente nesse artigo, como ilustra o Quadro 1.

Quadro 1: Relação de indicadores de desempenho sugeridos

\begin{tabular}{|c|c|c|c|c|c|}
\hline AUTORES & $\begin{array}{c}\text { Buarque } \\
\text { et al } \\
(\mathbf{2 0 0 3})\end{array}$ & $\begin{array}{c}\text { Amâncio } \\
\text { et al } \\
(2012)\end{array}$ & $\begin{array}{c}\text { Oliveira } \\
\text { Neto et al } \\
(2011)\end{array}$ & $\begin{array}{l}\text { Siluk et } \\
\text { al (2010) }\end{array}$ & $\begin{array}{r}\text { Ângelo } \\
(\mathbf{2 0 0 5})\end{array}$ \\
\hline \multicolumn{6}{|c|}{ DE CUSTOS } \\
\hline Controle de gastos com contratação de terceiros & $\mathbf{X}$ & & $\mathbf{X}$ & & \\
\hline Custo operacional total & $\mathbf{X}$ & & & $\mathbf{X}$ & \\
\hline Custos com atividades de transporte & $\mathbf{X}$ & & & & \\
\hline Custo de transporte como percentual de vendas & & & & & $\mathbf{X}$ \\
\hline Custo de frete por unidade expedida & & & & & $\mathbf{X}$ \\
\hline Percentual da mão de obra no custo total & $\mathbf{X}$ & & & & \\
\hline Pedidos cobrados corretamente & & & & $\mathbf{X}$ & \\
\hline Retorno sobre investimento & $\mathbf{X}$ & & & & \\
\hline \multicolumn{6}{|c|}{ DE TEMPO } \\
\hline Percentual de entregas fora do prazo & $\mathbf{X}$ & $\mathbf{X}$ & $\mathbf{X}$ & $\mathbf{X}$ & $\mathbf{X}$ \\
\hline \multicolumn{6}{|c|}{ Continuação Tabela 1} \\
\hline $\begin{array}{l}\text { Tempo entre pedido e recebimento das } \\
\text { mercadorias }\end{array}$ & $\mathbf{X}$ & $\mathbf{X}$ & $\mathbf{X}$ & $\mathbf{X}$ & $\mathbf{X}$ \\
\hline Tempo de descarga da carga & & & $\mathbf{X}$ & & \\
\hline Aderência ao agendamento de entrega & & & $\mathbf{X}$ & & \\
\hline Pedidos atendidos no prazo & $\mathbf{X}$ & & & & \\
\hline Tempo do ciclo de pedido & & & & & $\mathbf{X}$ \\
\hline \multicolumn{6}{|c|}{ DE QUALIDADE } \\
\hline Eficiência no recebimento & & $\mathbf{X}$ & & & \\
\hline Qualidade no atendimento & $\mathbf{X}$ & & & & \\
\hline Treinamento específico & & $\mathbf{X}$ & & & \\
\hline Não conformidades em transporte & & & & & $\mathbf{X}$ \\
\hline Motivo de reclamações & $\mathbf{X}$ & & & & \\
\hline $\begin{array}{l}\text { Tempo de espera para receber informações sobre } \\
\text { os serviços prestados }\end{array}$ & $\mathbf{X}$ & & & & \\
\hline Índice de roubos/furtos de carga & & & & $\mathbf{X}$ & $\mathbf{X}$ \\
\hline Incidências de avarias & & & $\mathbf{X}$ & $\mathbf{X}$ & $\mathbf{X}$ \\
\hline \multicolumn{6}{|c|}{ ACORDO DE NÍVEL DE SERVIÇO } \\
\hline Cumprimento das clausulas do contrato & & $\mathbf{X}$ & & & \\
\hline
\end{tabular}

Fonte: Adaptado de Sucena e Silva (2013) 


\section{WTEERFAET TECNOLLGGCA}

\section{RESULTADO E DISCUSSÃO}

Buarque et al. (2003), com base nas informações obtidas em seus estudos, concluíram que as empresas prestadoras de serviços de transporte deparam-se com dificuldades no levantamento de dados precisos para a avaliação dos serviços prestados.

A pesquisa bibliográfica, reúne diversas opiniões sobre o uso de indicadores de desempenho. Fica claro que independente a metodologia de embasamento na elaboração de um planejamento estratégico fundamentada na utilização de KPI (Key Performance Indicators), como podem ser chamados os indicadores chave de desempenho, é efetiva na busca constante por melhor performance e fundamental ferramenta de auxílio na tomada de decisão de gestores.

A terceirização das atividades de transporte rodoviário é uma forte tendência na busca por otimização de custos e processos, e segundo conclusão de Sucena e Silva (2013), traz melhor percepção de qualidade e agilidade junto aos clientes. E seguem completando que foi identificado a necessidade de atenção dos gestores, com relação aos terceirizados, para assegurar que através de ferramentas de avaliação e mensuração, haja maior garantia de que os serviços prestados atendam às necessidades e objetivos junto a empresa e seus clientes.

Baseado nos resultados obtidos, conclui-se que é essencial o monitoramento e avaliação de terceirizados para alcançar os objetivos empresariais. Sugerindo o uso de indicadores de desempenho como ferramenta auxiliadora na criação e desenvolvimento do plano estratégico logístico, assim como na criação de vantagem competitiva em busca da eficiência em processos de transporte de cargas utilizando o modal rodoviário.

Pois, o uso de KPI é uma excelente ferramenta estratégica e vem sendo incorporada por diversos autores e empresas, além de proporcionar a elaboração de planos de melhoria contínua em busca de oferecer, ao mercado, um alto nível de serviço ao cliente e garantir a qualidade em seus processos.

\section{CONSIDERAÇÕES FINAIS}

A próxima etapa da pesquisa consiste na elaboração de um modelo para análise de resultados de performance de uma empresa que atue no setor de serviços de fretes rodoviários na região de Bebedouro, SP. Para que em forma de estudo de caso sejam apresentados quantitativamente os benefícios possíveis elencados no resultada da pesquisa. 


\section{WTEERFAET TECNOLLGGCA}

Nessa investigação bibliográfica os objetivos e o problema de pesquisa foram atendidos, e os resultados servirão como base para estudo futuro que assumirá a forma de estudo de caso. Será realizado o levantamento de dados "reais" que permitirão a comparação entre a teoria e a prática.

\section{REFERÊNCIAS}

ÂNAGELO, L. B.; Indicadores de desempenho Logístico. Grupo de Estudos Logísticos, $2005 . \quad$ Capturado de <http://pessoal.utfpr.edu.br/anacristina/arquivos/A6\%20TextoIndicadores.pdf>. Disponível em: 23 out. 2012.

BARROS, M.; Terceirização logística no Brasil, 2009. Capturado de <http://www.ilos.com.br/web/terceirizacao-logistica-no-brasil/>. Disponível em: 02 mai. 2018.

BALLOU, R. H. Gerenciamento da cadeia de suprimentos/logística empresarial. 5.ed. Porto Alegre: Bookman, 2006.

BOWERSOX, D. J.; CLOSS, D. J.; COOPER, M. B. Supply Chain Logistics Management. 2a Edição. ed. Nova York: McGraw-Hill, 2007.

BUARQUE, R. C. S.; SILVA FILHO, J. C. G.; MIRANDA, L. C. Medição de Desempenho em Empresas de Transporte Rodoviário de Cargas: uma Investigação em Recife/PE, 2013. Disponível em: <http://eco.unne.edu.ar/contabilidad/costos/VIIIcongreso/281.doc>. Acesso em: 02 mai. 2018.

DIAS, M. A. Introdução a logística/fundamentos, práticas e integração. São Paulo: Atlas, 2017.

FLEURY, P. F.; WANKE, P.; FIGUEIREDO, K. F. Logística empresarial: a perspectiva brasileira. Coleção COPPEAD de Administração. São Paulo: Atlas, 2000.

KAPLAN, R. S.; NORTON, D. P. A Estratégia em Ação: Balanced Scorecard. 4.ed. Rio de Janeiro: Editora Campus. 1997.

GONÇALVES, P. S.; Administração de Materiais. Rio de Janeiro: Elsevier Editora Ltda, 2013.

MAZZA, L.; CORREIA, M. L. Indicadores de desempenho logístico pelas perspectivas do Balanced Scorcard., 2016. Disponível em: <http://www.tecnologistica.com.br/portal/artigos/72694/indicadores-de-desempenho-

logistico-pelas-perspectivas-do-balanced-scorecard/?\&\&\&\&desktop=1>. Acesso em: 02 mai. 2018.

MAXIMIANO, A. C. A. Teoria Geral da Administração. 2.ed. São Paulo: Editora Atlas, 2008. 
SUCENA, M. P.; SILVA, L. P. da. Relação entre teoria e prática para avaliação do desempenho no transporte rodoviário terceirizado de cargas, 2013. Disponível em: <http://www.inovarse.org/filebrowser/download/15727>. Acesso em: 02 mai. 2018. 